\title{
METHOD OF LINES TRANSPOSE: AN IMPLICIT SOLUTION TO THE WAVE EQUATION
}

\author{
MATTHEW CAUSLEY, ANDREW CHRISTLIEB, BENJAMIN ONG, \\ AND LEE VAN GRONINGEN
}

\begin{abstract}
We present a new method for solving the wave equation implicitly in one spatial dimension. Our approach is to discretize the wave equation in time, following the method of lines transpose, sometimes referred to as the transverse method of lines, or Rothe's method. We then solve the resulting system of partial differential equations using boundary integral methods.

Our algorithm extends to higher spatial dimensions using an alternating direction implicit (ADI) framework. Thus we develop a boundary integral solution that is competitive with explicit finite difference methods, both in terms of accuracy and speed. However, it provides more flexibility in the treatment of source functions and complex boundaries.

We provide the analytical details of our one-dimensional method herein, along with a proof of the convergence of our schemes in free space and on a bounded domain. We find that the method is unconditionally stable and achieves second order accuracy. Upon examining the discretization error, we derive a novel optimal quadrature method, which can be viewed as a Lax-type correction
\end{abstract}

\section{INTRODUCTION}

The wave equation is an important hyperbolic partial differential equation (PDE) that arises in acoustics, electromagnetics, and fluid dynamics. The focus of this paper is to define and analyze a new method for computing numerical solutions to the one-dimensional wave equations

$$
\begin{gathered}
v_{t t}=c^{2} v_{x x}, \quad x \in[-L, L], \quad t \geq 0 \\
v(x, 0)=f(x), \quad v_{t}(x, 0)=g(x), \quad v( \pm L, t)=0 \quad \text { or } \quad v_{x}( \pm L, t)=0 .
\end{gathered}
$$

We will consider the case of homogeneous boundary conditions, however, these ideas are easily extended to non-homogeneous Dirichlet and Neumann boundary conditions. Extensions to higher dimensions are discussed in Section 7.2

There are well established approaches to compute a numerical solution to the wave equation. One approach is to use separation of variables; that is, we assume a time harmonic solution $v(x, t)=\psi(x) e^{(i \omega t)}$, where $\omega$ is the temporal frequency. Then the amplitude satisfies the (oscillatory) Helmholtz equation $\left(\partial_{x x}+k^{2}\right) \psi=0$, where $k$ is the wave number, and $k^{2}=\omega^{2} / c^{2}$ is the dispersion relation. Boundary integral solutions for the Helmholtz equation in $\mathbb{R}^{2}$ and $\mathbb{R}^{3}$ can be computed efficiently using fast summation methods such as tree codes [10] or the fast multipole

Received by the editor January 30, 2012 and, in revised form, November 6, 2012 and March 8, 2013.

2010 Mathematics Subject Classification. 65N12, 65N40, 35L05.

Key words and phrases. Method of lines transpose, tranverse method of lines, implicit methods, boundary integral methods, alternating direction implicit methods, ADI schemes. 
method [2,5]. However, this approach is only useful for generating time harmonic solutions to the wave equation.

More generally, solutions are typically sought using the Method of Lines (MOL), in which $v\left(x_{i}, t\right)$ is treated as a function of time at a set of discrete spatial points $x_{i}$, and the resulting initial value problems (IVP) are solved in time. The spatial points are chosen according to some specific collocation method, such as Galerkin, finite difference or finite volume methods. Because the spatial derivatives are turned into algebraic equations, the MOL approach benefits from the extensive literature and numerical libraries for ODE solvers. Furthermore, the accuracy and stability of the MOL discretization for various families of PDEs has been thoroughly investigated and is well understood, e.g., [9, 15].

In addition to the MOL method, we can also proceed by first discretizing only in time, and finding a solution for the resulting Boundary Value Problems (BVPs). This approach is known as the Method of Lines Transpose $\left(\mathrm{MOL}^{T}\right)$, or sometimes the transverse method of lines. It has been sparsely considered [1,3, 7, 8, 11, 13, in part because the numerical solution to BVPs are more challenging, especially when the solution contains boundary layers.

However, there are several advantages to the $\mathrm{MOL}^{T}$ approach. For one, the resulting boundary value problem for the semi-discrete wave equation is now the modified (non-oscillatory) Helmholtz equation. The corresponding Green's function now exhibits exponential decay at infinity, rather than rapid oscillations. Recently, treecode [10] and FMM 44 algorithms have been developed for this kernel in $\mathbb{R}^{3}$, which is also known as the Yukawa or screened Coulomb potential, reducing the cost of evaluation from $O\left(N^{2}\right)$ to $O(N)$ or $O(N \log N)$.

Additionally, by formulating the problem as an integral solution, small cells can be incorporated near non-Cartesian boundaries without any loss of accuracy or rise of instabilities. Finally, the inclusion of point sources at arbitrary locations within the domain can be accurately computed, simply by convolution with the Green's function. These two latter points are distinct advantages over MOL approaches, and in light of recent fast summation algorithms, the $\mathrm{MOL}^{T}$ approach is increasingly more appealing.

In this paper, we formulate an implicit integral solution to equation (1.1) using $\mathrm{MOL}^{T}$ and inverting the modified Helmholtz operator. Our method will be based on the Yukawa potential in one spatial dimension, which is simply an exponential function. Thus, we develop a fast algorithm for obtaining boundary integral solutions, which is competitive with implicit finite difference methods in terms of computational complexity and accuracy, but inherits the aforementioned properties of incorporating small cells and arbitrary placement of sources. Furthermore, we can employ alternating direction implicit (ADI) methods to extend our algorithm to higher dimensions; a two-dimensional example is shown in Section 7 , and we reserve further discussion on this topic for another manuscript.

In Section 3 we will analyze these semi-discrete boundary integral solutions, and prove their consistency with solutions to the wave equation. Next, we present the fully discretized numerical algorithm in Section 4. We find that when the midpoint or trapezoidal rules are applied to perform quadrature on the boundary integral, then the leading order error term is not $O\left(\Delta x^{2}+\Delta t^{2}\right)$ as expected, but rather $O\left(\Delta x^{2}+(\Delta x / \Delta t)^{2}\right)$, so that as the step size and mesh are refined for a constant CFL number, the error is non-vanishing! Instead, we must have $\Delta t \sim \Delta x^{1-\epsilon}$, for 
some small positive value of $\epsilon$ in order to achieve convergence. This difficulty is circumvented by imposing a Lax-type correction to the leading order truncation error term, resulting in a novel quadrature method (4.7), which is optimal for our problem, and ensures convergence without any coupling between $\Delta t$ and $\Delta x$. When this optimal second order quadrature and first/second order time discretization is used, the leading order error is $O\left(\Delta x^{2}+\Delta t\right)$ or $O\left(\Delta x^{2}+\Delta t^{2}\right)$, respectively.

In order to prove convergence, we make use of the Lax-Richtmeyer equivalence theorem, which states that any consistent stable numerical algorithm will converge to the exact solution, provided the problem is Cauchy. Thus, in Section 5 we use Von Neumann stability analysis to prove stability of our algorithm. We perform this analysis for both the free-space problem and on a bounded domain. The latter result is of great importance for a boundary integral method, although stability proofs rarely address a bounded domain.

In addition to the discretization error, we analyze the phase error for sinusoidal solutions to the wave equation in Section 6. This section highlights the importance of the Lax-type correction, which dramatically reduces the phase error, in comparison to the trapezoidal quadrature scheme.

In Section 7 we present some numerical examples, comparing our method to other relevant and comparable methods for solving the wave equation. In Section 7.2 we extend our method to two and three spatial dimensions using ADI methods. In Section 8, we conclude the paper.

\section{Boundary integral solutions Using MOL ${ }^{T}$}

We will begin by applying the $\mathrm{MOL}^{T}$ to the wave equation (1.1). Below, we reserve $u^{n}=u^{n}(x)$ for the semi-discrete approximation to $v\left(x, t^{n}\right)$, the continuous solution of (1.1). When we consider collocation of the Laplacian term in various manners, two different classes of discretization schemes can be considered: dissipative and non-dissipative schemes.

2.1. Dissipative schemes. To construct the dissipative schemes, we first observe that the one-sided first and second order finite difference approximations to $v_{t t}^{n+1}=$ $v_{t t}\left(x, t^{n+1}\right)$ are:

$$
\begin{aligned}
& v_{t t}^{n+1}=\frac{v^{n+1}-2 v^{n}+v^{n-1}}{\Delta t^{2}}+O(\Delta t), \\
& v_{t t}^{n+1}=\frac{2 v^{n+1}-5 v^{n}+4 v^{n-1}-v^{n-2}}{\Delta t^{2}}+O\left(\Delta t^{2}\right) .
\end{aligned}
$$

If we evaluate the Laplacian term at time $t^{n+1}$, and make the substitution (2.1), we have a backward difference formula (BDF) scheme

$$
\frac{u^{n+1}-2 u^{n}+u^{n-1}}{\Delta t^{2}}=c^{2} u_{x x}^{n+1} \text {. }
$$

It will be shown in Section 3 that equation (2.3), along with the analogous approximation arising from the second order approximation (2.2) produces numerical dissipation. This effect may be desirable, for instance, when considering asymptotic preserving methods, for which $c \rightarrow \infty$ (here, $c$ is scaled by some characteristic time and length scale), and the hyperbolic nature of the equation transitions to an elliptic form. 
2.2. Purely dispersive schemes. An alternative implicit formulation follows from time-centering the derivative term. For example, if we use

$$
v_{x x}^{n}=\partial_{x x}\left(\frac{v^{n+1}+v^{n-1}}{2}\right)+O\left(\Delta t^{2}\right),
$$

then a second order accurate semi-discrete approximation to equation (1.1) is

$$
\frac{u^{n+1}-2 u^{n}+u^{n-1}}{(c \Delta t)^{2}}=\partial_{x x}\left(\frac{u^{n+1}+u^{n-1}}{2}\right) .
$$

In Section 3 we show this symmetric formulation removes numerical dissipation. Such purely dispersive schemes are more favorable for long time simulations, which circumvent the spurious effects of numerical diffusion.

2.3. Boundary integral solution. This work differs from other $\mathrm{MOL}^{T}$ methods in that we consider integral solutions to the boundary value problem, rather than utilizing finite difference or finite element methods for the spatial derivatives. Specifically, we solve the resulting BVP by obtaining the free space Green's function [6, 8, 11, and applying Green's theorem to obtain a boundary integral equation.

Observe that in both the dissipative (2.3) and non-dissipative (2.4) schemes, the modified Helmholtz equation is obtained, although it may act on $u^{n+1}$ alone, or some combination of $u^{n+1}, u^{n}$, and $u^{n-1}$. We therefore define the general Helmoltz operator

$$
\mathcal{L}_{\beta}[w](x):=\left(\partial_{x x}-\alpha^{2}\right) w(x), \quad \alpha=\frac{\sqrt{\beta}}{c \Delta t},
$$

and the corresponding Helmholtz equation

$$
\mathcal{L}_{\beta}[w](x)=-f_{\beta}(x),
$$

where $f_{\beta}(x)$ represents a general source function parameterized by $\beta$.

Equation (2.6) can be formally solved by inverting the Helmholtz operator. Using Green's theorem, the integral solution is obtained as the sum of a particular and a homogeneous solution, which incorporates boundary data.

$$
\begin{aligned}
w(x) & =\mathcal{L}_{\beta}^{-1}\left[-f_{\beta}(x)\right]=w_{p}(x)+w_{h}(x), \\
w_{p}(x) & =\int_{-L}^{L} f_{\beta}(y) G(x \mid y) d y, \\
w_{h}(x) & =\left[f_{\beta}(y) \partial_{y} G(x \mid y)-\partial_{y} f_{\beta}(y) G(x \mid y)\right]_{-L}^{L} .
\end{aligned}
$$

For the first order dissipative scheme (2.3) we set $w=u^{n+1}$ so that

$$
u^{n+1}(x)=\mathcal{L}_{1}^{-1}\left[-\alpha^{2}\left(2 u^{n}(x)-u^{n-1}(x)\right)\right] .
$$

The second order purely dispersive scheme (2.4) (with $w=u^{n+1}+u^{n-1}$ ) is

$$
u^{n+1}(x)+u^{n-1}(x)=\mathcal{L}_{2}^{-1}\left[-2 \alpha^{2} u^{n}(x)\right] .
$$

Other schemes can be derived in a similar fashion. In $\mathbb{R}^{1}$, the free space Green's function for the modified Helmholtz equation is

$$
G(x \mid y)=-\frac{e^{-\alpha|x-y|}}{2 \alpha} .
$$


Notice that in both dissipative and non-dissipative schemes, the right-hand side of the Helmholtz equations (2.8) and (2.9) are proportional to $\alpha^{2}$. Hence, we need only consider the quadrature of an infinite or finite convolution integral

$$
I[u](x)=\alpha \int_{-\infty}^{\infty} u(y) e^{-\alpha|x-y|} d y, \quad J[u](x)=\alpha \int_{-L}^{L} u(y) e^{-\alpha|x-y|} d y,
$$

where $u$ represents a linear combination of $u^{n}$ and $u^{n-1}$, according to either (2.3) or (2.4). It follows that

$$
w_{p}(x)=J\left[\frac{-f_{\beta}(x)}{\alpha^{2}}\right]
$$

2.4. Homogeneous boundary conditions. The homogeneous term $w_{h}$ can be understood as a correction to enforce the boundary conditions. In one dimension, it can be written as a linear combination of the homogeneous solutions to the modified Helmholtz equation. Thus, we replace (2.7c) with the ansatz

$$
w_{h}(x)=c_{1} e^{-\alpha(L-x)}+c_{2} e^{-\alpha(L+x)},
$$

and find $c_{1}$ and $c_{2}$ upon imposing given boundary conditions.

Specifically, for homogeneous Dirichlet conditions, we have

$$
0=\left.w\right|_{x= \pm L}=\left.\left(w_{p}+w_{h}\right)\right|_{x= \pm L} .
$$

Solving the resulting linear system for the unknowns $c_{1}$ and $c_{2}$ gives

$$
w_{h}^{D}(x)=-\frac{\sinh (\alpha(L-x))}{\sinh (2 \alpha L)} w_{p}(-L)-\frac{\sinh (\alpha(L+x))}{\sinh (2 \alpha L)} w_{p}(L) .
$$

Alternatively, for homogeneous Neumann conditions, we have

$$
0=\left.w^{\prime}\right|_{x= \pm L}=\left.\left(w_{p}^{\prime}+w_{h}^{\prime}\right)\right|_{x= \pm L} .
$$

Solving the resulting linear system for the unknowns $c_{1}$ and $c_{2}$ gives

$$
w_{h}^{N}(x)=\frac{\cosh (\alpha(L-x))}{\sinh (2 \alpha L)} \frac{w_{p}^{\prime}(-L)}{\alpha}-\frac{\cosh (\alpha(L+x))}{\sinh (2 \alpha L)} \frac{w_{p}^{\prime}(L)}{\alpha} .
$$

The hyperbolic functions appearing in the coefficients can vary by several orders of magnitude, and so we rescale them to avoid potential numerical instabilities. If we neglect terms that are less than $e^{-2 \alpha L}$ in magnitude, then the Dirichlet conditions take on a simpler form

$$
w_{h}^{D}(x) \approx-e^{-\alpha(L+x)} w_{p}(-L)-e^{-\alpha(L-x)} w_{p}(L) .
$$

Likewise, homogeneous Neumann conditions are given approximately by

$$
w_{h}^{N}(x) \approx e^{-\alpha(L+x)} \frac{w_{p}^{\prime}(-L)}{\alpha}-e^{-\alpha(L-x)} \frac{w_{p}^{\prime}(L)}{\alpha} .
$$

These simpler boundary conditions will be accurate to order $\epsilon$ provided $c \Delta t \leq$ $2 L / \log (1 / \epsilon)$. For double precision computations, we may safely use equations (2.15) or (2.16) provided that a wave traveling with speed $c$ does not traverse the domain in less than 20 time steps. In computations of practical interest this is usually not a limitation, and so we use these effective boundary conditions without reservation. 


\section{AnAlysis OF THE SEMI-Discrete SOLUTION}

We begin by establishing consistency for the semi-discrete integral solutions presented in Section 2. We obtain results for the general free space problem, and then a specific result for homogeneous boundary conditions. Hence we prove the following:

Lemma 3.1. Let $u^{n+1}(x)$ be the semi-discrete $M O L^{T}$ solution (2.8). Then $u^{n+1}(x)$ will be consistent with $O(\Delta t)$. If $u^{n+1}(x)$ is the semi-discrete $M O L^{T}$ solution (2.9), then it will be consistent with $O\left(\Delta t^{2}\right)$.

3.1. Consistency in free space. Let $v$ be the the exact solution satisfying (1.1) on an infinite domain. The global truncation error for the semi-discrete non-dissipative scheme (2.4) is

$$
\tau^{n}(x)=\frac{v^{n+1}-2 v^{n}+v^{n-1}}{(c \Delta t)^{2}}-\partial_{x x}\left(\frac{v^{n+1}+v^{n-1}}{2}\right)=\frac{-5 \Delta t^{2}}{12 c^{2}} v_{t t t t}(x, \zeta) .
$$

We now show that this agrees to leading order with the truncation error for the integral solution in free space (2.4),

$$
u^{n+1}(x)=I\left[u^{n}\right](x)-u^{n-1}(x),
$$

where $I\left[u^{n}\right](x)$ is as in equation (2.11). We define a modified truncation error

$$
\tilde{\tau}^{n}(x)=\frac{v^{n+1}+v^{n-1}-I\left[v^{n}\right](x)}{(c \Delta t)^{2}},
$$

which depends on the nature of the integral solution.

Lemma 3.2. For $u \in C^{2 p}(\mathbb{R})$,

$$
I[u](x)=2 \sum_{k=0}^{p-1}\left(\frac{1}{\alpha}\right)^{2 k} \partial_{x}^{2 k} u(x)+\frac{2}{\alpha^{2 p}} \partial_{x}^{2 p} u(\xi) .
$$

Proof. We note that

$$
I[u](x)=-2 \alpha^{2} \int G(x \mid y) u(y) d y, \quad \partial_{x x} G(x \mid y)=\alpha^{2} G(x \mid y)+\delta(x-y) .
$$

Thus

$$
\begin{aligned}
\partial_{x x} I[u](x) & =-2 \alpha^{2} \int \partial_{x x}^{2} G(x \mid y) u(y) d y \\
& =-2 \alpha^{2} \int\left(\alpha^{2} G(x \mid y)+\delta(x-y)\right) u(y) d y=\alpha^{2} I[u](x)-2 \alpha^{2} u(x) .
\end{aligned}
$$

Performing integration by parts twice (and making use of the fact that $G$ vanishes at infinity),

$$
\partial_{x x} I[u](x)=I\left[u_{x x}\right](x) .
$$

Equating both expressions,

$$
I[u](x)=2 u(x)+\frac{1}{\alpha^{2}} I\left[u_{x x}\right](x) .
$$

This argument can be iterated up to order $2 p$, and applying the integral mean value theorem, we obtain the desired result. 
We now make use of this result, truncating at $p=2$, to give

$$
I\left[v^{n}\right](x)=2 v^{n}(x)+\frac{2}{\alpha^{2}} v_{x x}^{n}(x)+\frac{2}{\alpha^{4}} v_{x x x x}^{n}(\xi) .
$$

The truncation error (3.1) then becomes

$$
\begin{aligned}
\tilde{\tau}^{n} & =\frac{1}{(c \Delta t)^{2}}\left(v^{n+1}+v^{n-1}-I\left[v^{n}\right]\right) \\
& =\frac{1}{(c \Delta t)^{2}}\left(v^{n+1}+v^{n-1}-2 v^{n}\right)-\frac{1}{(c \Delta t)^{2}}\left(\frac{2}{\alpha^{2}} v_{x x}^{n}(x)+\frac{2}{\alpha^{4}} v_{x x x x}^{n}(\xi)\right) \\
& =\frac{1}{c^{2}}\left(v_{t t}+\frac{\Delta t^{2}}{12} v_{t t t t}(x, \eta)\right)-\left(v_{x x}+\frac{(c \Delta t)^{2}}{2} v_{x x x x}\left(\xi, t_{n}\right)\right) \\
& =\frac{\Delta t^{2}}{12 c^{2}} v_{t t t t}(x, \eta)-\frac{\Delta t^{2}}{2 c^{2}} v_{t t t t}\left(\xi, t_{n}\right)=\frac{-5 \Delta t^{2}}{12 c^{2}} v_{t t t t}^{n}(\xi, \zeta)
\end{aligned}
$$

where we have expanded the terms $v^{n \pm 1}$ in a Taylor series about $t^{n}$, and that

$$
v_{t t t t}=c^{2} v_{x x t t}=c^{4} v_{x x x x} .
$$

The same ideas may now be applied to the first order dissipative free space solver (2.3). However, the expansion is now about $t^{n+1}$, and the semi-discrete solution is given by

$$
u^{n+1}(x)=I\left[u^{n}-\frac{1}{2} u^{n-1}\right](x)
$$

while the truncation error is

$$
\tau^{n+1}=\frac{1}{(c \Delta t)^{2}}\left(v^{n+1}-I\left[v^{n}-\frac{1}{2} v^{n-1}\right]\right) .
$$

Using Lemma 3.1 (with $p=1$ ), a Taylor expansion about $t_{n+1}$ gives

$$
\begin{aligned}
\tau^{n+1} & =\frac{1}{(c \Delta t)^{2}}\left(v^{n+1}-2 v^{n}+v^{n-1}-(c \Delta t)^{2} \partial_{x x}\left(2 v^{n}-v^{n-1}\right)(\xi)\right) \\
& =\frac{1}{c^{2}}\left(v_{t t}^{n+1}-\Delta t v_{t t t}^{n+1}\right)-v_{x x}^{n+1}+O\left(\Delta t^{2}\right)=-\frac{\Delta t}{c^{2}} v_{t t t}^{n+1}(x, \zeta),
\end{aligned}
$$

and thus the integral solution is first order accurate in time.

3.2. Consistency with boundary conditions. Now we consider the consistency of the semi-discrete solution when homogeneous Dirichlet conditions are implemented. For the purely dispersive solver (2.4), the truncation error is

$$
\tau^{n}=\frac{1}{(c \Delta t)^{2}}\left(v^{n+1}+v^{n-1}-J\left[v^{n}\right](x)\right),
$$

where

$$
J\left[v^{n}\right](x)=\left(-2 \alpha^{2}\right) \int_{-L}^{L} G_{D}(x \mid y) v^{n}(y) d y .
$$

The Green's function $G_{D}$, which satisfies homogeneous Dirichlet boundary conditions, is found by expanding the definitions of $w_{p}( \pm L)$, so that

$$
G_{D}(x \mid y)=\frac{e^{-\alpha|x-y|}}{-2 \alpha}+\frac{\sinh (\alpha(L+x)) e^{-\alpha(L-y)}+\sinh (\alpha(L-x)) e^{-\alpha(L+y)}}{2 \alpha \sinh (2 \alpha L)}
$$


Note that written in this manner, the boundary integrals vanish identically, since both $G_{D}$ and $v$ vanish at $x= \pm L$. We omit the truncation error analysis for the homogeneous Neumann problem, but record the corresponding Green's function

$$
G_{N}(x \mid y)=\frac{e^{-\alpha|x-y|}}{-2 \alpha}+\frac{\cosh (\alpha(L+x)) e^{-\alpha(L-y)}-\cosh (\alpha(L-x)) e^{-\alpha(L+y)}}{2 \alpha \sinh (2 \alpha L)} .
$$

We also observe that at $x= \pm L, v^{n}$ and all even spatial derivatives vanish,

$$
\lim _{x \rightarrow \pm L} \partial_{x}^{2 m} v(x, t)=\lim _{x \rightarrow \pm L}\left(\frac{1}{c} \partial_{t}\right)^{2 m} v(x, t)=\left(\frac{1}{c} \partial_{t}\right)^{2 m} v( \pm L, t)=0 .
$$

The order of the limits and the derivatives can be swapped as long as the $2 m$-th derivatives of $v^{n}$ exist and are continuous.

We proceed as before with repeated use of integration by parts. In analog to the free space problem, we have the following:

Lemma 3.3. Suppose $u(x) \in C^{2 p}([-L, L])$ satisfies $\partial_{x}^{2 k} u(x= \pm L)=0$, for $k=$ $0,1, \ldots, p$. Then

$$
J[u](x)=2 \sum_{k=0}^{p-1}\left(\frac{1}{\alpha}\right)^{2 k} \partial_{x}^{2 k} u(x)+\frac{2}{\alpha^{2 p}} q(x) \partial_{x}^{2 p} u(\xi),
$$

where $q(x)=\frac{1}{2} \int_{-L}^{L} G_{D}(x \mid y) d y$.

Proof. If we take the Laplacian of $J[u](x)$, we find

$$
\begin{aligned}
\partial_{x x} J[u](x) & =-2 \alpha^{2} \int_{-L}^{L} \partial_{x x} G_{D}(x \mid y) u(y) d y \\
& =-2 \alpha^{2} \int_{-L}^{L}\left(\alpha^{2} G_{D}(x \mid y)+\delta(x-y)\right) u(y) d y=\alpha^{2} J[u](x)-2 \alpha^{2} u(x) .
\end{aligned}
$$

Integrating by parts gives $\partial_{x x} J[u](x)=J\left[u_{x x}\right](x)$ for $x \in(-L, L)$. Hence,

$$
J[u](x)=2 u(x)+\frac{1}{\alpha^{2}} J\left[u_{x x}\right](x) .
$$

Upon iterating this argument up to order $2 p$, we obtain the desired result. The last remainder term involving $q(x)$ is an application of the integral mean value theorem.

The global truncation error can now be obtained:

$$
\begin{aligned}
\tau^{n} & =\frac{1}{(c \Delta t)^{2}}\left(v^{n+1}+v^{n-1}-J\left[v^{n}\right]\right) \\
& =\frac{1}{(c \Delta t)^{2}}\left(v^{n+1}+v^{n-1}-\left[2 v^{n}+(c \Delta t)^{2} v_{x x}^{n}+q(x) \frac{(c \Delta t)^{4}}{2} v_{x x x x}^{n}(\xi)\right]\right) \\
& =\frac{1}{c^{2}}\left(v_{t t}^{n}+\frac{\Delta t^{2}}{12} v_{t t t t}^{n}+O\left(\Delta t^{4}\right)\right)-v_{x x}^{n}-q(x) \frac{(c \Delta t)^{2}}{2} v_{x x x x}^{n}(\xi) \\
& =\frac{(1-6 q(x))(c \Delta t)^{2}}{12} v_{x x x x}(\xi, \eta) .
\end{aligned}
$$

A rather involved calculation gives

$$
q(x)=\frac{1}{2} \int_{-L}^{L} G_{D}(x, y) d y=1-\frac{\sinh (\alpha(L+x))}{\sinh (2 \alpha L)}-\frac{\sinh (\alpha(L-x))}{\sinh (2 \alpha L)} .
$$


Notice that $q( \pm L)=0$ and $q(x) \approx 1$ away from the boundary, whenever $\alpha L$ is large. This is to be expected, as the error term should vanish identically at the boundary (the function is imposed exactly there), and approximately recover the free space solution in the interior of the domain. Hence, the semi-discrete dissipative scheme on a finite domain is second order accurate in time, and is very similar to that found for the free space problem.

Similar proofs will hold for homogeneous Neumann boundary conditions, as well as for the first order dissipative scheme.

\section{ONE-DIMENSIONAL FORMULATION}

In this section we detail our numerical approach in one spatial dimension. In Section 7.2 we will extend this approach to higher dimensions using ADI splitting.

The proceeding analysis will be used to prove the convergence of our numerical scheme, which will follow from the Lax-Richtmeyer equivalence theorem. Specifically, we prove the following:

Theorem 4.1. Let $v\left(x_{j}, t_{n+1}\right)$ be the continuous solution at $\left(x_{j}, t_{n+1}\right)$ to the wave equation (1.1) with homogeneous Dirichlet or Neumann boundary conditions. If $u_{j}^{n+1}(x)$ is the discrete first order dissipative $M O L^{T}$ solution (4.9), with the corresponding Dirichlet (4.16) or Neumann (4.18) boundary conditions, and the optimal quadrature rule (4.7) is applied, then $u_{j}^{n+1}$ will be accurate with $v\left(x_{j}, t_{n+1}\right)$ of $O\left(\Delta t+\Delta x^{2}\right)$. If the second order non-dissipative $M O L^{T}$ scheme (4.10) is used instead, then $u_{j}^{n+1}$ will be accurate with $v\left(x_{j}, t_{n+1}\right)$ of $O\left(\Delta t^{2}+\Delta x^{2}\right)$. Both methods for obtaining a numerical solution are stable for $0 \leq t \leq T, T>0$, and thus convergent.

4.1. Fully discrete solution. Consider the spatial discretization of the particular (2.7b) and homogeneous (2.7c) solutions in our boundary integral solution (2.7a). We first develop a quadrature rule for (2.11). Next we apply it to homogeneous Dirichlet and Neumann conditions.

Let the real line be partitioned into subintervals of length $\Delta x$, and define $x_{j}=$ $j \Delta x$. Evaluating both $u(x)$ and $I[u]$ at the midpoint values $x=x_{j-1 / 2}$ yields

$$
\begin{aligned}
I[u]\left(x_{j-1 / 2}\right) & =\alpha \int_{-\infty}^{\infty} u(y) e^{-\alpha\left|y-x_{j-1 / 2}\right|} d y \\
& \approx \sum_{i=-\infty}^{\infty} u_{i-1 / 2}\left(\alpha \int_{x_{i-1}}^{x_{i}} e^{-\alpha\left|y-x_{j-1 / 2}\right|} d y\right) .
\end{aligned}
$$

The remaining integral can be evaluated analytically. Hence,

$$
I_{j-1 / 2}=2 a_{0} u_{i-1 / 2}+2 a_{1} \sum_{i \neq j} e^{-|j-i| \nu} u_{i-1 / 2},
$$

where $\nu=\alpha \Delta x$ and

$$
a_{0}=1-e^{-\frac{\nu}{2}}, \quad a_{1}=\sinh \left(\frac{\nu}{2}\right) .
$$

Similarly, we may use the trapezoidal rule to obtain a quadrature, with $u(x)$ and $I[u]$ both evaluated at the values $x=x_{i}$. Upon replacing $u$ with a linear interpolant and evaluating the resulting integrals analytically we find

$$
I_{j}=2 a_{0} u_{j}+2 a_{1} \sum_{i \neq j} e^{-|j-i| \nu} u_{i}
$$


where now

$$
a_{0}=1-\frac{1-e^{-\nu}}{\nu}, \quad a_{1}=\frac{2}{\nu} \sinh ^{2}\left(\frac{\nu}{2}\right) .
$$

In both quadrature rules, there is a relation between $a_{0}$ and $a_{1}$,

$$
a_{0}=1-\frac{2 a_{1}}{e^{\nu}-1},
$$

which is implied by insisting that the rule be exact for constant functions $u$.

The quadrature error can be found by expanding $u(x)$ in a Taylor series about $x_{j}$ (or $x_{j-1 / 2}$ for the midpoint rule). We utilize (4.4), and obtain

$$
\begin{aligned}
I_{j} & =2 u_{j}+2 a_{1} \sum_{k=1}^{\infty} e^{-k \nu}\left(u_{j+k}+u_{j-k}-2 u_{j}\right) \\
& =2 u_{j}+4 a_{1} \sum_{k=1}^{\infty} e^{-k \nu}\left(\frac{(k \Delta x)^{2}}{2} u_{j}^{\prime \prime}+\frac{(k \Delta x)^{4}}{24} u^{(4)}(\xi)\right) \\
& =2 u_{j}+a_{1}\left(4 \sum_{k=1}^{\infty} k^{2} e^{-k \nu}\right) \frac{\Delta x^{2}}{2} u_{j}^{\prime \prime}+a_{1}\left(4 \sum_{k=1}^{\infty} k^{4} e^{-k \nu}\right) \frac{\Delta x^{4}}{24} u^{(4)}(\xi) \\
& =2 u_{j}+\tilde{a}_{1}\left(\frac{\Delta x^{2}}{2} u_{j}^{\prime \prime}+\left(1+\frac{3}{\sinh ^{2}\left(\frac{\nu}{2}\right)}\right) \frac{\Delta x^{4}}{24} u^{(4)}(\xi)\right),
\end{aligned}
$$

where

$$
\tilde{a}_{1}=\frac{a_{1}}{\tanh \left(\frac{\nu}{2}\right) \sinh ^{2}\left(\frac{\nu}{2}\right)} .
$$

Comparing with (3.2), the quadrature error is

$$
I[u]\left(x_{j}\right)-I_{j}=\left(\frac{1}{\alpha^{2}}-\tilde{a}_{1} \frac{\Delta x^{2}}{2}\right) u_{j}^{\prime \prime}+O\left(\Delta x^{4}+\left(\frac{1}{\alpha^{4}}\right)\right) .
$$

This quadrature error appears as an additional term in the truncation error (3.7), which is subsequently scaled by a factor of $(c \Delta t)^{2}$; thus the fully discrete truncation error contains a term that is $O\left(\Delta x^{2} /(c \Delta t)^{2}\right)=O\left(\nu^{2}\right)$. We emphasize that this error term will remain $O(1)$ whenever $\Delta t=O(\Delta x)$, even if the time step is refined.

4.2. A Lax-type correction. In order to overcome this difficulty, we apply a Laxtype correction, which will raise the accuracy of the quadrature rule, and eliminate this term from the truncation error. For simplicity, we treat the parameters $a_{0}$ and $a_{1}$ from equation (4.2) as free parameters, rather than defining them using a quadrature rule. We now impose (4.4) as before, but use the remaining free parameter to impose that the leading order term of the quadrature error (4.6) vanish. In doing so, we find a quadrature rule of the form (4.2), with

$$
a_{0}=1-\frac{2}{e^{\nu}+1}\left(\frac{2}{\nu} \sinh \left(\frac{\nu}{2}\right)\right)^{2}, \quad a_{1}=\left(\frac{2}{\nu} \sinh \left(\frac{\nu}{2}\right)\right)^{2} \tanh \left(\frac{\nu}{2}\right) .
$$

We will refer to this quadrature rule as an optimal two-parameter quadrature rule for the integrals (2.11) in the sense that it uniquely reduces the size of the truncation error (3.3). 
Lemma 4.2. The fully discrete solution $u_{j}^{n}$, obtained by applying the midpoint and trapezoidal rules to equation (2.7) will be accurate of order $O\left(\Delta t^{\beta}+\nu^{2}\right)$, where $\beta=1$ if equation (2.8) is solved, and $\beta=2$ if (2.9) is solved. The optimal quadrature (4.7) obtains accuracy of $O\left(\Delta t^{\beta}+\Delta x^{2}\right)$.

4.3. Discrete treatment of boundary conditions. Returning the focus of the discussion to a finite domain, we examine the sum (4.2), and incorporate the boundary conditions. Let $x_{j}=-L+j \Delta x$ for $j=0, \ldots, N$, where $\Delta x=2 L / N$. On a finite domain, the quadrature rule takes the form

$$
J_{j}=a_{0}\left(2 u_{j}-u_{0} d^{j}-u_{N} d^{N-j}\right)+2 a_{1}\left(\sum_{k=1}^{j} d^{k} u_{j-k}+\sum_{k=1}^{N-j} d^{k} u_{j+k}\right),
$$

where $d=e^{-\alpha \Delta x}$, and the new terms are due to endpoint corrections. Define the solution vector $\bar{u}^{n}=\left[u^{n}\left(x_{0}\right), \ldots, u^{n}\left(x_{N}\right)\right]^{T}$. An analogous vector will be defined for the midpoint rule, collocated at the semi-grid points $x_{j-1 / 2}$ for $j=1, \ldots N$. We now interpret (4.8) as a the product of the solution vector with a discrete convolution matrix $A_{p}$. In direct analog to the particular (2.7b) and homogeneous (2.7c) solutions, define the total discrete convolution matrix as $A=A_{p}+A_{h}$. Then the first order dissipative scheme corresponding to equation (2.3) is formulated as

$$
\bar{u}^{n+1}=A\left(2 \bar{u}^{n}-\bar{u}^{n-1}\right) .
$$

The second order non-dissipative scheme corresponding to equation (2.4) is

$$
\bar{u}^{n+1}=2 A \bar{u}^{n}-\bar{u}^{n-1} .
$$

Since we are solving the wave equation discretely, the action of the matrix $A$ is to propagate waves to the left and right, with speed $c$, and so we can interpret $A$ as the propagation matrix. The structure of $A$, which we will detail below, makes our fast algorithm possible; both the particular and homogeneous matrix vector products can be computed in $O(N)$ operations.

To build the discrete convolution matrix, define the vectors

$$
\begin{aligned}
\bar{v}_{ \pm}=e^{-\alpha(L \pm \bar{x})}, \quad \bar{w}_{ \pm}^{D} & =\frac{\bar{v}_{\mp}-e^{-2 \alpha L} \bar{v}_{ \pm}}{1-e^{-4 \alpha L}}=\frac{1}{1-d^{2 N}}\left(\bar{v}_{\mp}-d^{N} \bar{v}_{ \pm}\right), \\
\bar{w}_{ \pm}^{N} & =\frac{\bar{v}_{\mp}+e^{-2 \alpha L} \bar{v}_{ \pm}}{1-e^{-4 \alpha L}}=\frac{1}{1-d^{2 N}}\left(\bar{v}_{\mp}+d^{N} \bar{v}_{ \pm}\right),
\end{aligned}
$$

where $\bar{x}=(-L,-L+\Delta x, \ldots, L-\Delta x, L)^{T}$ corresponds to the grid points for the trapezoidal and (Lax) optimal rules (and the semi-gridpoints for the midpoint rule), and $d=e^{-\nu}=e^{-\alpha \Delta x}$. The vectors $\bar{w}_{ \pm}$are used in the implementation of either Dirichlet or Neumann boundary conditions, according to their superscripts. We first use equation (4.8) to determine the form of $A_{p}$. For the midpoint rule, the particular matrix is given by

$$
A_{p}=\left(a_{0}-a_{1}\right) I+a_{1} T
$$

where $I$ is the $N \times N$ identity matrix and $T$ is a symmetric Toeplitz matrix whose first row (and column) is given by $v_{+}$. Note that $T$ is defined solely in terms of the 
parameter $d$, as

$$
T=\left(\begin{array}{ccccc}
1 & d & \ldots & & d^{N-1} \\
d & 1 & & & \\
\vdots & & \ddots & & \vdots \\
& & & 1 & d \\
d^{N-1} & & \ldots & d & 1
\end{array}\right)
$$

The trapezoidal and (Lax) optimal quadratures are similar, but $T$ will now be of size $(N+1) \times(N+1)$, and the endpoint corrections, which can be written as rank-one updates, will be included; thus

$$
A_{p}=\left(a_{0}-a_{1}\right) I+a_{1} T-\frac{a_{0}}{2}\left(\bar{v}_{+} \otimes \bar{e}_{1}+\bar{v}_{-} \otimes \bar{e}_{N+1}\right)
$$

where $\bar{e}_{1}=(1,0, \ldots 0)^{T}$ and $\bar{e}_{N+1}=(0, \ldots, 0,1)^{T}$.

The boundary matrices follow from discretization of (2.13) and (2.14). Omitting the details, we find

$$
\begin{aligned}
A_{h}^{D}= & -a_{1}\left[\bar{w}_{-}^{D} \otimes\left(\bar{v}_{+}-\bar{e}_{1}\right)+\bar{w}_{+}^{D} \otimes\left(\bar{v}_{-}-\bar{e}_{N+1}\right)\right] \\
& -\frac{a_{0}}{2}\left[\left(\bar{w}_{-}^{D}-d^{N} \bar{w}_{+}^{D}\right) \otimes \bar{e}_{1}+\left(\bar{w}_{+}^{D}-d^{N} \bar{w}_{-}^{D}\right) \otimes \bar{e}_{N+1}\right], \\
A_{h}^{N}= & a_{1}\left[\bar{w}_{-}^{N} \otimes\left(\bar{v}_{+}-\bar{e}_{1}\right)+\bar{w}_{+}^{N} \otimes\left(\bar{v}_{-}-\bar{e}_{N+1}\right)\right] \\
& +\frac{a_{0}}{2}\left[\left(\bar{w}_{-}^{N}-d^{N} \bar{w}_{+}^{N}\right) \otimes \bar{e}_{1}+\left(\bar{w}_{+}^{N}-d^{N} \bar{w}_{-}^{N}\right) \otimes \bar{e}_{N+1}\right] .
\end{aligned}
$$

As mentioned in Section 2, the homogeneous Dirichlet boundary conditions undergo a substantial simplification provided terms that are $O\left(d^{N}=e^{-2 \alpha L}\right)$ are small. We will first make use of the identities

$$
\bar{w}_{ \pm}^{D}=\bar{v}_{\mp}-d^{N} \bar{w}_{\mp}^{D}, \quad \bar{w}_{ \pm}^{N}=\bar{v}_{\mp}+d^{N} \bar{w}_{\mp}^{N},
$$

and subsequently neglect all terms that are $O\left(d^{N}\right)$ in the boundary matrices (4.14) and (4.15). After some additional algebra, the full discrete convolution matrix for the Dirichlet problem is

$$
\begin{aligned}
A^{D}= & \left(a_{0}-a_{1}\right)\left(I-e_{1} \otimes e_{1}-e_{N+1} \otimes e_{N+1}\right) \\
& +a_{1}\left(T-\bar{w}_{-}^{D} \otimes \bar{v}_{+}-\bar{w}_{+}^{D} \otimes \bar{v}_{-}\right) \\
\approx & \left(a_{0}-a_{1}\right)\left(I-e_{1} \otimes e_{1}-e_{N+1} \otimes e_{N+1}\right)+a_{1}\left(T-\bar{v}_{+} \otimes \bar{v}_{+}-\bar{v}_{-} \otimes \bar{v}_{-}\right)
\end{aligned}
$$

while the Neumann matrix is

$$
\begin{aligned}
A^{N} & =\left(a_{0}-a_{1}\right) I+a_{1}\left(T+\bar{w}_{-}^{N} \otimes\left(\bar{v}_{+}-\bar{e}_{1}\right)+\bar{w}_{+}^{N} \otimes\left(\bar{v}_{-}-\bar{e}_{N+1}\right)\right) \\
& \approx\left(a_{0}-a_{1}\right) I+a_{1}\left(T+\bar{v}_{+} \otimes\left(\bar{v}_{+}-\bar{e}_{1}\right)+\bar{v}_{-} \otimes\left(\bar{v}_{-}-\bar{e}_{N+1}\right)\right) .
\end{aligned}
$$

The matrix $A$ is set to (4.16) or (4.18) depending on the boundary conditions, although in practice the approximations (4.17) and (4.19), respectively, are used for actual computation.

\section{Stability}

We now prove the stability of our fully discrete schemes (4.9) and (4.10) and complete the convergence proof of Theorem 4.1. 
Lemma 5.1. The fully discrete solution $u_{j}^{n}$, obtained with (4.10), with A given by (4.16) or (4.18) will be stable for $0 \leq t \leq T$, for any $T>0$, and any choice of $\Delta x$ and $\Delta t$. If $u_{j}^{n}$ is obtained with (4.9), with $A$ given by (4.13), then it will be stable for $0 \leq t \leq T$, for any $T>0$ and any choice of $\Delta x$ and $\Delta t$.

Remark 5.2. The bound we find below does not guarantee stability for the dissipative method (4.9), for a narrow range of $\nu$. Our numerical solutions have exhibited no instabilities, and evaluation of the true eigenvalues over the narrow range of $\nu$ shows that they have magnitude less than 1 .

Our proof utilizes Von Neumann stability analysis, and a special result for the eigenvalues of the Toeplitz matrix (4.12). We will establish stability for these schemes below by studying the eigenvalues of $A_{p}$ given by (4.11), and $A=A_{p}+A_{h}$. Since $A_{h}$ will be exponentially small away from the boundaries, the main part of our work will focus on analyzing the eigenvalues of $A_{p}$.

5.1. The Eigenvalues of $A_{p}$. We now make a crucial observation which allows us to make several analytical remarks about the stability of our algorithm. The inverse of our Toeplitz matrix $T$ in equation (4.12) is given by

$$
T^{-1}=\frac{1}{1-d^{2}}\left(\begin{array}{ccccc}
1+d^{2} & -d & & & \\
-d & 1 & & & \\
& \ddots & \ddots & \ddots & \\
& & & 1 & -d \\
& & & -d & 1+d^{2}
\end{array}\right)
$$

This observation makes it possible to use a result of Yueh and Cheng [14, in which the eigenvalues for tridiagonal matrices with perturbed corners are found. This establishes that the eigenvalues of $T$ are given by

$$
\tau_{k}=\frac{1-d^{2}}{1+d^{2}+2 d \cos \left(\theta_{k}\right)}, \quad 1 \leq k \leq N,
$$

where $\theta_{k}$ is a root of

$$
\sin ((N+1) \theta)+2 d \sin (N \theta)+d^{2} \sin ((N-1) \theta)=0, \quad \theta \in(0, \pi) .
$$

The spectrum of $T$ is easily bounded by letting $\theta_{k}=0, \pi$, and after some algebraic manipulation and making use of $d=e^{-\nu}$,

$$
\tanh \left(\frac{\nu}{2}\right)<\tau_{k}<\operatorname{coth}\left(\frac{\nu}{2}\right)
$$

Lemma 5.3. The eigenvalues $\lambda_{k}$ of the matrix $A_{p}$ given by equation (4.11) are contained in the interval $(0,1)$.

Proof. Observing the relationship between $A_{p}$ and $T$, the eigenvalues $\lambda_{k}$ are related to $\tau_{k}$ by

$$
\lambda_{k}=\left(a_{0}-a_{1}\right)+a_{1} \tau_{k}=1-a_{1}\left(\operatorname{coth}\left(\frac{\nu}{2}\right)-\tau_{k}\right)
$$

where we have used (4.4), and several hyperbolic trigonometric identities. We can now bound the eigenvalues, and after several more identities we find that

$$
1-\frac{a_{1}}{\sinh \left(\frac{\nu}{2}\right) \cosh \left(\frac{\nu}{2}\right)}<\lambda_{k}<1
$$


If we evaluate these expressions for the midpoint, trapezoidal and optimal quadratures we find that in all cases, $0<\lambda_{k}<1$, which proves the lemma.

Lemma 5.4. The matrix $A_{p}$ is symmetric positive definite, and therefore diagonalizable.

Proof. The symmetry of $A_{p}$ follows from that of $T$. Positive definiteness follows from the previous lemma.

5.2. Stability of the dissipative scheme with $A=A_{p}$. We now prove the stability of equations (4.9) and (4.10), with $A=A_{p}$ (i.e., $A_{h}=0$ ). Following the standard approach of Von Neumann, we make the ansatz $\bar{u}^{n}=\mu^{n} \bar{u}^{0}$ for $n \geq 0$, and find the amplification factors $\mu$. Since we have a multistep scheme, there will be $2 N$ roots of the Von Neumann polynomial. However, since we have shown that $A_{p}$ is a symmetric positive definite matrix, we know that there exists an orthonormal matrix $Q$ such that $A_{p}=Q \Lambda Q^{T}$, where $\Lambda$ is the diagonal matrix with entries given by (5.1). Thus, if we define $\bar{v}=Q^{T} \bar{u}^{0}$, and multiply equations (4.9) and (4.10) by $Q$,

$$
\begin{aligned}
\mu^{n+1} \bar{v} & =\left(2 \mu^{n}-\mu^{n-1}\right) \Lambda \bar{v}, \\
\mu^{n+1} \bar{v} & =\left(2 \mu^{n} \Lambda-\mu^{n-1} I\right) \bar{v} .
\end{aligned}
$$

Since these latter forms are expressed as the determinant of a diagonal matrix, the Von Neumann polynomial can be written in factored form, and the amplification factors of (5.2) $\mu_{k}^{ \pm}$will be determined in pairs by the expressions

$$
\mu_{k}^{2}-2 \lambda_{k} \mu_{k}+\lambda_{k}=0, \quad 1 \leq k \leq N,
$$

and similarly, for

$$
\mu_{k}^{2}-2 \lambda_{k} \mu_{k}+1=0, \quad 1 \leq k \leq N .
$$

The dissipative scheme will thus have amplification factors given by

$$
\mu_{k}^{ \pm}=\lambda_{k} \pm i \sqrt{\lambda_{k}-\lambda_{k}^{2}}
$$

Since $\lambda_{k} \in(0,1)$, we see immediately that the amplification factors form complex conjugate pairs. Further, since each $\theta_{k}$ is distinct, this means that for each $k$, the roots of the Von Neumann polynomial will also be distinct. If we further show that $\left|\mu_{k}^{ \pm}\right| \leq 1$, then we will have shown stability of the numerical scheme. Taking the modulus of the roots, we have

$$
\left|\mu_{k}^{ \pm}\right|=\sqrt{\lambda_{k}^{2}+\lambda_{k}-\lambda_{k}^{2}}=\sqrt{\lambda_{k}}<1 .
$$

Thus, the first order system will be stable and since $\left|\mu_{k}^{ \pm}\right|<1$, dissipative.

Likewise, the roots of the non-dissipative scheme are given as

$$
\mu_{k}^{ \pm}=\lambda_{k} \pm i \sqrt{1-\lambda_{k}^{2}} .
$$

This time, the modulus of each complex conjugate pair is

$$
\left|\mu_{k}^{ \pm}\right|=\sqrt{\lambda_{k}^{2}+1-\lambda_{k}^{2}}=1 .
$$

Thus, since the eigenvalues are all distinct, the method is stable and, since $|\mu|=1$, the scheme is non-dissipative. 
5.3. Stability with $A=A_{p}+A_{h}$. In this section, we turn our attention to the Dirichlet boundary matrix $A=A^{D}$, from equation (4.16). We treat the matrix $A_{h}^{D}$, given by equation (4.14) perturbatively, and look for the eigenvalues of $A$, denoted $\lambda+\delta$, where $\lambda$ is an eigenvalue of $A_{p}$. Stability will follow if we can show that $|\mu| \leq 1$, where $\mu$ is now given by

$$
\mu^{2}-2(\lambda+\delta) \mu+\lambda+\delta=0
$$

in the dissipative scheme and

$$
\mu^{2}-2(\lambda+\delta) \mu+1=0
$$

in the non-dissipative scheme. Note that the boundary corrections are such that the first and last columns, (as well as the rows) of $A^{D}$ are identically zero, which means that we can omit the terms in equation (4.14) involving $e_{1}$ and $e_{N+1}$, and the corresponding zero eigenvalues. Stability will hold provided $\lambda+\delta \in[-1,1]$ for the non-dissipative scheme, which can be shown to be the case using Gershgorin's theorem. However, for the dissipative scheme, we require the more restrictive range $\lambda+\delta \in[-1 / 3,1]$ to ensure stability. Below, we will make use of the Bauer-Fike theorem.

Theorem 5.5 (Bauer-Fike). Let $A$ be a diagonalizable matrix, and $Q$ the nonsingular eigenvector matrix such that $A=Q \Lambda Q^{-1}$, where $\Lambda$ is the diagonal matrix containing the eigenvalues of $A$. Then for the matrix $A+E$, with eigenvalues $\lambda+\delta$, and where $E$ is an arbitrary perturbation matrix, the following holds

$$
|\delta| \leq \kappa(Q)\|E\|
$$

where $\|\cdot\|$ denotes the 2 -norm, and $\kappa(\cdot)$ is the condition number.

We will apply this theorem with $A$ set to the middle $(N-1) \times(N-1)$ entries (i.e., non-zero rows and columns) of the free space matrix $A_{p}$ and $E$ the corresponding portion of $A_{h}^{D}$. Since $A_{p}$ is a real symmetric positive definite matrix (hence $Q$ is orthonormal), we have $\kappa(Q)=1$. Thus,

$$
|\delta| \leq\left\|A_{h}^{D}\right\|
$$

and so we restrict our attention to the norm of $A_{h}^{D}$, given by equation (4.14), (again, after omitting the terms involving $e_{1}$ and $e_{N+1}$ ). Since it is given entirely in terms of two vectors, $A_{h}^{D}$ is at most rank two. Specifically, we have [12] that the eigenvalues of $A_{h}^{D}$ are given by

$$
\lambda_{ \pm}=\frac{\operatorname{Tr}\left(A_{h}^{D}\right) \pm \operatorname{Tr}\left(J A_{h}^{D}\right)}{2}
$$

where $\operatorname{Tr}(\cdot)$ denotes the trace, and $J$ is the counter-identity matrix, with ones along the anti-diagonal. In particular, we note that $J v_{ \pm}=v_{\mp}$. Notice also that $A_{h}^{D}$ is symmetric; therefore, the 2-norm of the matrix is equal to its spectral radius (i.e., the absolute value of the maximum eigenvalue)

$$
\left\|A_{h}^{D}\right\|=\left|\frac{\operatorname{Tr}\left(A_{h}^{D}\right)+\operatorname{Tr}\left(J A_{h}^{D}\right)}{2}\right| .
$$


This value can be computed using dot products; observe that

$$
\begin{aligned}
& \operatorname{Tr}\left(\bar{v}_{ \pm} \otimes \bar{v}_{ \pm}\right)=\bar{v}_{ \pm} \cdot \bar{v}_{ \pm}=\sum_{k=1}^{N-1} e^{-2 \alpha\left(L \pm x_{k}\right)}=\frac{d^{2}}{1-d^{2}}\left(1-d^{2 N}\right), \\
& \operatorname{Tr}\left(\bar{v}_{ \pm} \otimes \bar{v}_{\mp}\right)=\bar{v}_{ \pm} \cdot \bar{v}_{\mp}=\sum_{k=1}^{N-1} e^{-2 \alpha L}=(N-1) d^{N}
\end{aligned}
$$

which follows from $\nu=\alpha \Delta x, 2 L=N \Delta x$, and $d=e^{-\nu}$. Additionally, $J w_{ \pm}=w_{\mp}$, and so we have

$$
\begin{aligned}
|\delta| \leq\left\|A_{h}^{D}\right\| & =\frac{a_{1}}{2} \operatorname{Tr}\left[\left(\bar{w}_{+}^{D}+\bar{w}_{-}^{D}\right) \otimes\left(\bar{v}_{+}+\bar{v}_{-}\right)\right] \\
& =\frac{a_{1}}{2} \frac{1-d^{N}}{1-d^{2 N}} \operatorname{Tr}\left[\left(\bar{v}_{+}+\bar{v}_{-}\right) \otimes\left(\bar{v}_{+}+\bar{v}_{-}\right)\right] \\
& =a_{1}\left(1-d^{N}\right)\left(\frac{d^{2}}{1-d^{2}}+\frac{d^{2 N}}{1-d^{2 N}}(N-1)\right)=a_{1} c(N, d) .
\end{aligned}
$$

In Table 1, the maximum value (over $N$ ) of the bound is given for various values of $\nu$; the values for the optimal and trapezoidal rules were identical to 4 digits of precision. The largest eigenvalue of $A_{h}^{D}$ is approximately 0.3361 , which is slightly larger than $1 / 3$. Thus, while our analysis cannot guarantee stability for the dissipative scheme, it more than does for the non-dissipative scheme. However, we stress that this bound is loose, and that in the indicated region the full eigenvalues $\lambda+\delta$ have been computed, and are less than 1 in magnitude. Thus, we conclude that the method is stable. To further validate this statement, numerical simulations using values of $N$ and $\nu$ corresponding to potential instabilities nonetheless produce stable convergent solutions.

TABLE 1. Bound for the perturbation of the free space eigenvalues

\begin{tabular}{|c|c|c|c|}
\hline$\nu$ & $\max |\delta|$ & $\nu$ & $\max |\delta|$ \\
\hline 0.001 & 0.3361 & 0.008 & 0.3342 \\
\hline 0.002 & 0.3358 & 0.016 & 0.3319 \\
\hline 0.004 & 0.3353 & 0.032 & 0.3275 \\
\hline
\end{tabular}

\section{DISPERSION}

We now analyze the phase error of our free space approximation. The continuous dispersion relation results from looking at sinusoidal solutions of the wave equation, $v(x, t)=e^{i(k x-\omega t)}$, which gives $\omega^{2}=c^{2} k^{2}$. We now analyze the semi-discrete and fully discrete dispersion relations, and define the phase error for the dissipative and non-dissipative schemes. We will only examine the free space problem here.

6.1. Semi-discrete schemes. For the semi-discrete equation we define $u^{n}(x)=$ $\hat{u} e^{i k x-i \tilde{\omega}(n \Delta t)}$, where $\tilde{\omega}$ denotes the discrete temporal frequency. Plugging this ansatz into the non-dissipative scheme (2.9), we get

$$
\left(e^{-i \tilde{\omega} \Delta t}+e^{i \tilde{\omega} \Delta t}\right) \hat{u} e^{i k x-i \tilde{\omega}(n \Delta t)}=\left(\alpha \int_{-\infty}^{\infty} e^{-\alpha|x-y|} e^{i k y} d y\right) \hat{u} e^{-i \tilde{\omega}(n \Delta t)} .
$$


We cancel the common term to get

$$
\cos (\tilde{\omega} \Delta t)=\frac{\alpha}{2} \int_{-\infty}^{\infty} e^{-\alpha|x-y|-i k(x-y)} d y=\frac{\alpha}{2} \int_{0}^{\infty} e^{-\alpha z}\left(e^{i k z}+e^{-i k z}\right) d z
$$

after the change of variables $z=y-x$. The right-hand side can be viewed as a Laplace transform of $\cos k z$, and this expedites the evaluation of the integral. Thus

$$
\cos (\tilde{\omega} \Delta t)=\frac{\alpha^{2}}{\alpha^{2}+k^{2}}=1-s
$$

where

$$
s=\frac{z^{2}}{1+z^{2}}, \quad z=\frac{k}{\alpha} .
$$

This is the semi-discrete analog to the continuous dispersion relation. To avoid aliasing, we are only interested in wave numbers in the region $0 \leq \tilde{\omega} \Delta t \leq \pi$. The phase error can then be defined as

$$
\Phi(\Delta t)=\left|\frac{\tilde{\omega}}{k c}-1\right| .
$$

For a fixed frequency $k$, we can analyze the convergence of this approximation using a Taylor series approximation for small $z=k / \alpha=k c \Delta t / \sqrt{2}$. First, we make use of trigonometric identities in (6.2) to obtain

$$
\sin ^{2}\left(\frac{\tilde{\omega} \Delta t}{2}\right)=\frac{s}{2}=\frac{1}{2}\left(\frac{z^{2}}{1+z^{2}}\right) .
$$

Now using the expansion for arcsin, we get

$$
\tilde{\omega}=\frac{2}{\Delta t} \arcsin \left(\frac{z}{\sqrt{2\left(1+z^{2}\right)}}\right)=k c\left(1-\frac{5(k c \Delta t)^{2}}{24}+O\left(\Delta t^{4}\right)\right)
$$

from which it follows that the phase error is second order.

For the dissipative scheme, we define $u^{n}$ as before, and eventually obtain $e^{-i \tilde{\omega} \Delta t}=$ $\left(2-e^{i \tilde{\omega} \Delta t}\right)(1-s)$, where $s$ is defined by (6.3). From this expression we isolate $\tilde{\omega}$ by solving a quadratic polynomial for $\lambda=e^{i \tilde{\omega} \Delta t}$. The quadratic equation now admits complex solutions $\lambda=1 \pm i k c \Delta t$. Thus, the temporal frequencies will be complex (dissipative),

$$
\tilde{\omega}=\frac{1}{i \Delta t} \log (1+i k c \Delta t)=k c\left(1-\frac{i k c \Delta t}{2}+O\left(\Delta t^{2}\right)\right),
$$

and the phase error is first order.

6.2. Fully discrete schemes. Next we focus our attention on the fully discrete form of the non-dissipative scheme (2.9). The only modification to equation (6.1) will be that the integral is replaced with an infinite sum. Let $u_{j}^{n}=\hat{u} e^{i(k j \Delta x-n \hat{\omega} \Delta t)}$, where now $\hat{\omega}$ denotes the fully discrete temporal frequency. We make use of (4.2), along with (4.4) to simplify the evaluation of the infinite sum. After cancellation of the common term, the fully discrete dispersion relation is

$$
\cos (\hat{\omega} \Delta t)=1-\frac{2 a_{1}}{e^{\nu}-1}+a_{1} \sum_{m=1}^{\infty} e^{-k m \nu}\left(e^{i k m \Delta x}+e^{-i k m \Delta x}\right)=1-\sigma
$$


where after some algebra we find the discrete analog to (6.3):

$$
\sigma=a_{1} \operatorname{coth}\left(\frac{\nu}{2}\right)\left(\frac{\zeta^{2}}{1+\zeta^{2}}\right), \quad \zeta=\frac{\sin \left(\frac{k \Delta x}{2}\right)}{\sinh \left(\frac{\nu}{2}\right)} .
$$

Notice that since $\nu=\alpha \Delta x, \zeta \approx z$ for small $k \Delta x$ and $\nu$. As in the semi-discrete analysis, we study the phase for a fixed frequency $k$. After some additional algebraic manipulation, we arrive at

$$
\sin ^{2}\left(\frac{\hat{\omega} \Delta t}{2}\right)=\frac{\sigma}{2}=\frac{a_{1}}{2} \operatorname{coth}\left(\frac{\nu}{2}\right)\left(\frac{\zeta^{2}}{1+\zeta^{2}}\right) .
$$

Since we are interested in implicit methods, the relevant inequality is $\Delta x<c \Delta t$, and so we consider the limit of the phase error for $\Delta x, \Delta t \rightarrow 0$, with $\nu$ a small, fixed quantity. If we assume that $k \Delta x \ll 1$, then it follows that $\omega \Delta t \ll 1$ as well, and the dispersion relation reduces to

$$
\hat{\omega} \Delta t \approx k \Delta x \sqrt{\frac{\tilde{a}_{1}}{2}}+O\left((k \Delta x)^{2}\right),
$$

where $\tilde{a}_{1}$ is defined in equation (4.5), and applies to each type of quadrature considered. After making use of $\nu=\alpha \Delta x$, the phase error satisfies

$$
\Phi(\nu, \Delta x, \Delta t)=\left|\frac{\hat{\omega}}{k c}-1\right| \approx\left|\sqrt{\tilde{a}_{1} \frac{\nu^{2}}{4}}+O\left((k \Delta x)^{2}\right)-1\right| .
$$

Perusal of the quadrature schemes we have considered reveals that the midpoint (4.1) and trapezoidal (4.3) rules will produce a phase error that is $O\left(\nu^{2}\right)$, whereas for the optimal quadrature (4.7), the fraction inside the radical of the phase error reduces exactly to unity, and the phase error is now properly second order. In Figure 1, the phase error is greatly reduced with the optimal quadrature when compared to the trapezoidal quadrature for the fully discrete scheme. In particular, the error in the static limit is zero in the former case, not in the latter. The fully discrete dispersion relation for the dissipative scheme is

$$
e^{-i \hat{\omega} \Delta t}=\left(2-e^{i \hat{\omega} \Delta t}\right)(1-\sigma)
$$

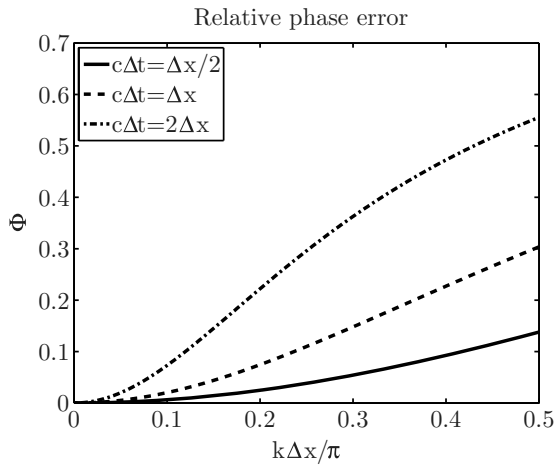

(a) Optimal quadrature (4.7)

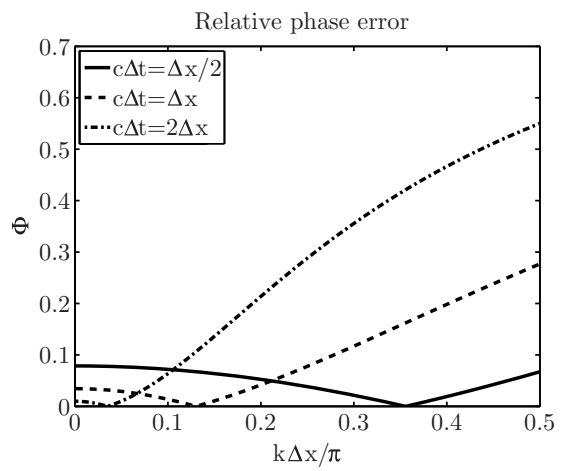

(b) Trapezoidal rule 4.3.

FiguRE 1. Phase error comparison 
where $\sigma$ is defined by (6.5). The dispersion relation is a quadratic polynomial in $\lambda=e^{(i \hat{\omega} \Delta t)}$, with solution $\lambda=1 \pm i \sqrt{\frac{\sigma}{1-\sigma}}$. Thus

$$
\hat{\omega}=\frac{1}{i \Delta t} \log \left(1 \pm i \sqrt{\frac{a_{1} \operatorname{coth}\left(\frac{\nu}{2}\right) \zeta^{2}}{1+\zeta^{2}+a_{1} \operatorname{coth}\left(\frac{\nu}{2}\right) \zeta^{2}}}\right) .
$$

Finally, if we fix $\nu$ and $k$ and let $\Delta x \rightarrow 0$, the static limit of the first order phase error is given by

$$
\lim _{\Delta x \rightarrow 0} \tilde{\Phi}(\nu, \Delta x, \Delta t)=\left|\frac{\hat{\omega}}{k c}-1\right| \approx\left|\sqrt{\tilde{a}_{1} \frac{\nu^{2}}{4}}+O\left((k \Delta x)^{2}\right)-1\right|,
$$

which is exactly the same limit as for the non-dissipative scheme.

\section{Numerical Results}

7.1. One-dimensional results. In this section we present a refinement study of the numerical schemes (4.9) and (4.10). The wave equation is computed on the unit interval, with wave speed $c=2$, up to a time $t=T=1$, and zero Dirichlet conditions are imposed. For the initial condition, we prescribe a Gaussian

$$
v(x, 0)=e^{-144\left(x-\frac{1}{2}\right)^{2}}, \quad v_{t}(x, 0)=0,
$$

which will be initially of negligible amplitude at the boundaries, and will have the exact solution

$$
v(x, t)=\frac{1}{2}\left(e^{-144\left(x-c t-\frac{1}{2}\right)^{2}}+e^{-144\left(x+c t-\frac{1}{2}\right)^{2}}\right) .
$$

The problem is discretized so that the ratio $c \Delta t / \Delta x$ is held fixed at 10 in the first order dissipative scheme, and 20 in the second order non-dissipative scheme. Note that in both cases, this quantity is well beyond the CFL stability limit imposed on explicit finite difference schemes. The spatial step $\Delta x=1 / N$ is varied in the range corresponding to $N \in[50,12800]$, and $\Delta t$ is scaled accordingly. The relative error is computed using the norm

$$
\|u(x, t)\|=\left\|\left(\|u(x, t)\|_{L^{2}[0,1]}\right)\right\|_{L^{\infty}[0, T]}
$$

and the results are shown in Figure 2, Notice that in the first order scheme, the midpoint and trapezoidal methods perform as well as the optimal quadrature rule, since the range of $\Delta t$ is such that $\Delta t>\nu^{2}$, where again $\nu=\frac{\sqrt{2} \Delta x}{c \Delta t}$, and so the error due to spatial discretization is not observed. This is in contrast to the second order scheme, which shows the superiority of the optimal quadrature. The error in the midpoint and trapezoidal rules will not decrease beyond $O\left(\nu^{2}\right)$ even as we refine $\Delta t$ and $\Delta x$.

In addition to studying the convergence of the scheme, we also present results pertaining to the numerical efficiency of our algorithm. We solve an initial boundary value problem with homogeneous Dirichlet conditions, and initial values given by $v(x, 0)=\sin (\pi x)$, and $v_{t}(x, 0)=0$. The numerical solution is obtained in two ways, with exactly the same discrete parameters. First, we illustrate our $\mathrm{MOL}^{T}$ approach using the time centered scheme (4.10), where the propagation matrix 


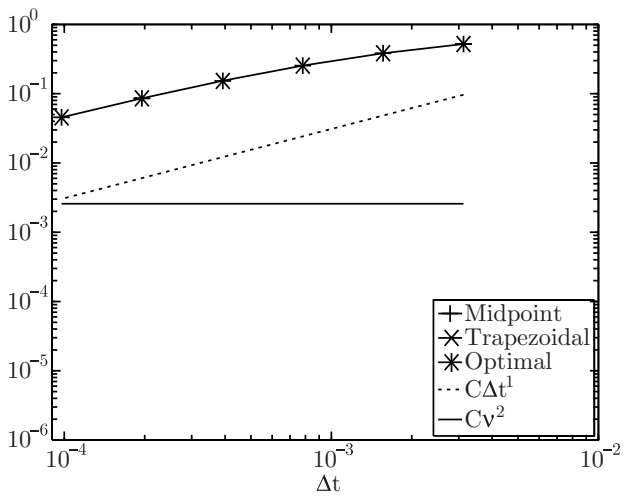

(a) First order scheme (4.9)

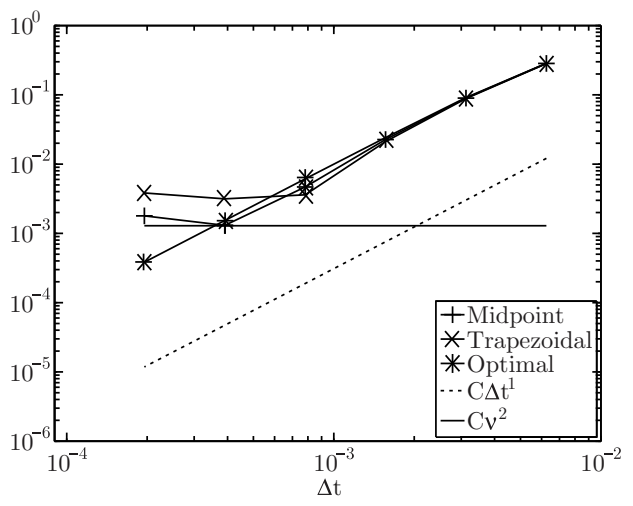

(b) Second order scheme 4.10)

Figure 2. Convergence of numerical schemes

(4.16) is defined using the optimal quadrature rule (4.7). For comparison, we also use the same fully discrete stencil, but apply finite differences in space. That is,

$$
\left(\frac{\delta_{x}^{2}}{\Delta x^{2}}-2 \frac{1}{(c \Delta t)^{2}}\right)\left(u_{j}^{n+1}+u_{j}^{n-1}\right)=-\frac{4}{(c \Delta t)^{2}} u_{j}^{n},
$$

where $\delta_{x}^{2}$ is the standard second order spatial finite difference operator. This latter scheme is solved using the Thomas algorithm, which is an efficient $O(N)$ tridiagonal solver. The mesh spacing is fixed at $\Delta x=0.01$, corresponding to 200 spatial points over the interval $[-1,1]$, and the CFL number is initially set to 1 and successively doubled. The final computation time $T=10$ is held fixed, and so the number of time steps is successively halved. We therefore expect the total computation time to be similarly reduced by a factor of two, which can be observed in Table 2, and confirms the linear scaling of both algorithms. We also report the relative $L^{2}$ error at the final time and, as can be observed, the results are comparable. We point out that while the initial start up time of the $\mathrm{MOL}^{T}$ is greater than that of the implicit finite difference algorithm, they are both negligible in terms of the total run time. All quantities shown are taken as the average over 400 realizations.

TABLE 2. Efficiency of method of lines transpose

\begin{tabular}{|c|c|c|c|c|c|c|}
\hline & \multicolumn{3}{|c|}{ Implicit FD } & \multicolumn{3}{c|}{ MOL $^{T}$} \\
\hline CFL & Rel. Err. & Setup (s) & Total (s) & Rel. Err. & Setup (s) & Total (s) \\
\hline 1 & $3.0 \times 10^{-5}$ & $3.6 \times 10^{-6}$ & $2.4 \times 10^{-2}$ & $2.2 \times 10^{-5}$ & $2.2 \times 10^{-4}$ & $2.0 \times 10^{-2}$ \\
\hline 2 & $3.7 \times 10^{-4}$ & $2.9 \times 10^{-6}$ & $1.3 \times 10^{-2}$ & $3.3 \times 10^{-4}$ & $1.1 \times 10^{-4}$ & $1.0 \times 10^{-2}$ \\
\hline 4 & $5.4 \times 10^{-3}$ & $3.7 \times 10^{-6}$ & $6.3 \times 10^{-3}$ & $5.2 \times 10^{-3}$ & $1.1 \times 10^{-4}$ & $5.1 \times 10^{-3}$ \\
\hline
\end{tabular}

7.2. Extension to higher dimensions. Thus far we have developed a numerical approach for solving the one-dimensional wave equation implicitly. Since the matrices (4.16) and (4.18) are comprised of a diagonal matrix, a Toeplitz matrix and several rank-one updates, their product with a vector can be computed in $\mathrm{O}(\mathrm{N})$ operations. We can make use of this result in higher dimensions by performing ADI 
splitting. Observe that the Helmholtz operator (2.5)

$$
\left(\nabla^{2}-\alpha^{2}\right)=-\alpha^{2}\left(1-\frac{1}{\alpha^{2}} \frac{\partial^{2}}{\partial x^{2}}\right)\left(1-\frac{1}{\alpha^{2}} \frac{\partial^{2}}{\partial y^{2}}\right)\left(1-\frac{1}{\alpha^{2}} \frac{\partial^{2}}{\partial z^{2}}\right)+O\left(\frac{1}{\alpha^{2}}\right)
$$

which shows that each spatial component of the Laplacian can be factored, resulting in three $\mathbb{R}^{1}$ problems. The error is $O\left(1 / \alpha^{2}\right)=O\left(c^{2} \Delta t^{2}\right)$, which is the same order as the truncation error. Thus, the fast algorithm developed can be used to obtain fully three-dimensional boundary integral solutions. More details about this algorithm will be reported in a separate work.

For now we show several examples of our method. First, we utilize the ADI scheme presented to generate sinusoidal modes on a rectangular geometry $(x, y) \in$ $\left[0, L_{x}\right] \times\left[0, L_{y}\right]$. The initial condition is

$$
u(x, y, 0)=\sin \left(\frac{2 \pi x}{L_{x}}\right) \cos \left(\frac{2 \pi x}{L_{y}}\right), \quad u_{t}(x, y, 0)=0,
$$

and we impose homogeneous Dirichlet conditions at $x=0, L_{x}$, with homogeneous Neumann conditions in $y=0, L_{y}$.
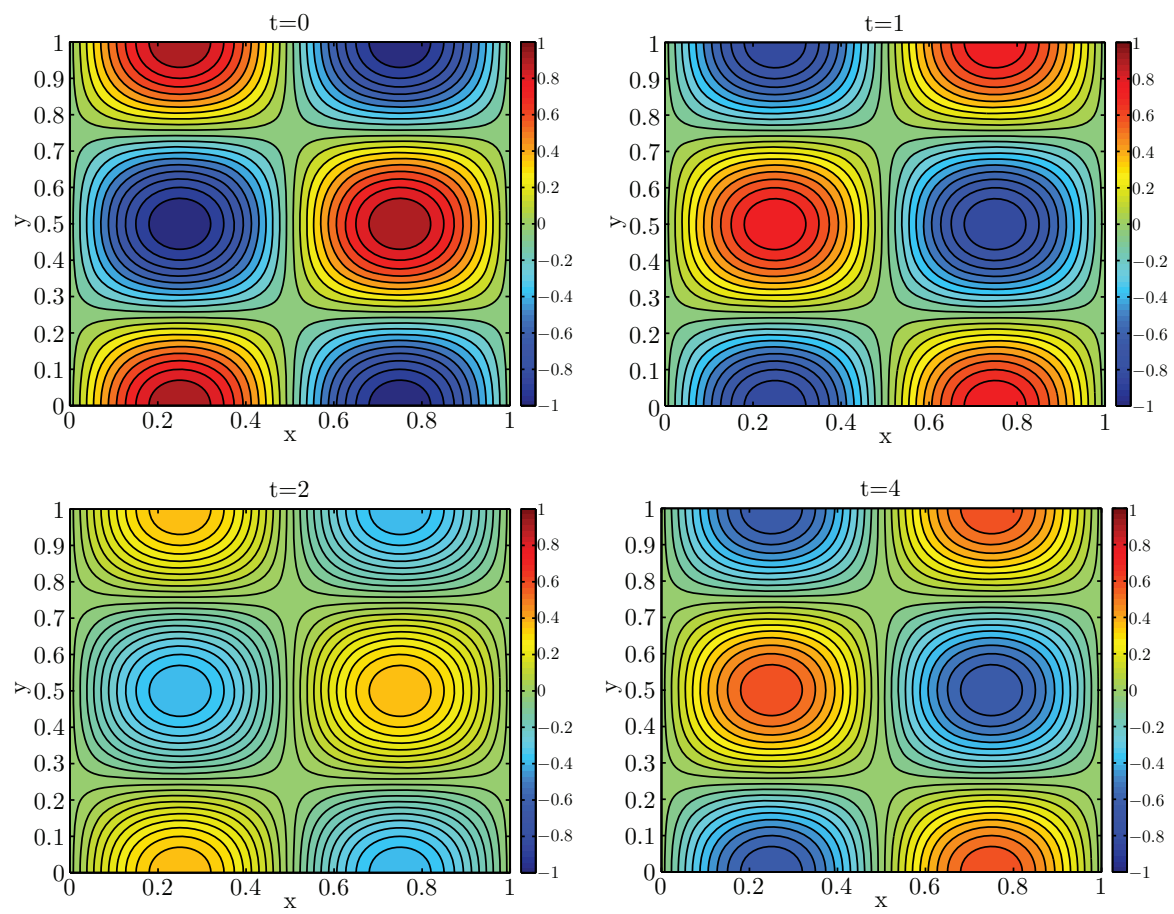

Figure 3. Plots of the $(1,1)$ mode on a square domain

Several plots of the solution are shown in Figure 3. Here we have taken $L_{x}=$ $L_{y}=1, \Delta x=\Delta y=1 / 256$, and $\Delta t=1 / 80$. In Table 3 , the second order rate of convergence is confirmed. The error is computed as in equation (7.1).

Next, we show the power of using an integral method over a differential method with the following classical example of a vibrating mode. We impose homogeneous 
TABLE 3. Convergence of the $(1,1)$ mode on a square domain

\begin{tabular}{|c|c|c|}
\hline$\Delta t$ & Error & Rate \\
\hline $1.000 \times 10^{-1}$ & $3.425 \times 10^{-1}$ & $* * *$ \\
$5.000 \times 10^{-2}$ & $1.046 \times 10^{-1}$ & 1.711 \\
$2.500 \times 10^{-2}$ & $2.754 \times 10^{-2}$ & 1.926 \\
$1.250 \times 10^{-2}$ & $7.014 \times 10^{-3}$ & 1.973 \\
$6.250 \times 10^{-3}$ & $1.771 \times 10^{-3}$ & 1.986 \\
$3.125 \times 10^{-4}$ & $4.573 \times 10^{-3}$ & 1.954 \\
\hline
\end{tabular}

Dirichlet conditions on a circle of radius $R$, with

$$
u(x, y, 0)=J_{0}\left(z_{2} \frac{\sqrt{x^{2}+y^{2}}}{R}\right), \quad u_{t}(x, y, 0)=0,
$$

where $J_{0}$ is the Bessel function of the first kind of order 0 , and $z_{2} \approx 5.5201$ is its second zero. The exact solution is then given by $u(x, y, t)=u(x, y, 0) \cos \left(z_{2} c t\right)$, and the numerical solution is shown for several times in Figure 4. To perform the computation, the unit circle $(R=1)$ was embedded inside a square mesh of width $\Delta x=\Delta y=0.01$, and the solution was truncated to include only those points which fall within (or on) the circle. The points that are used in the $x$ and $y$ sweep are shown in Figures 5(a) and 5(b) respectively.
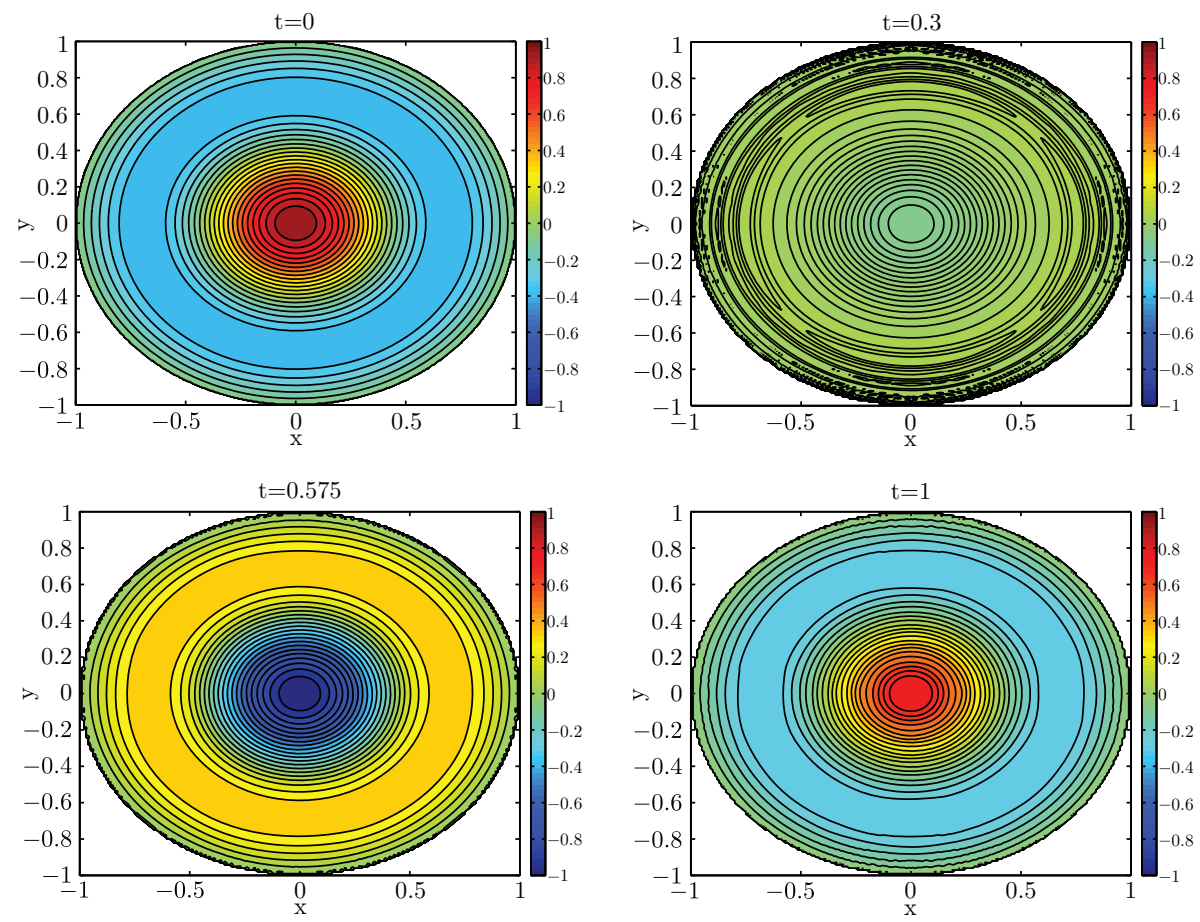

Figure 4. Plots of the $(2,0)$ mode on a circular domain 


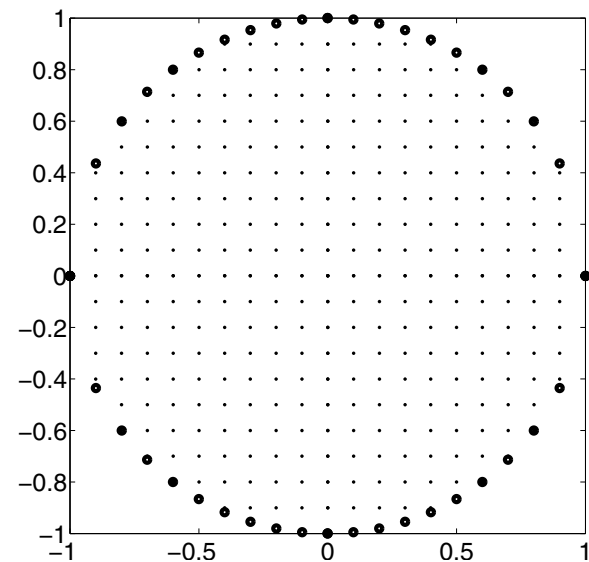

(a) $x$ sweep

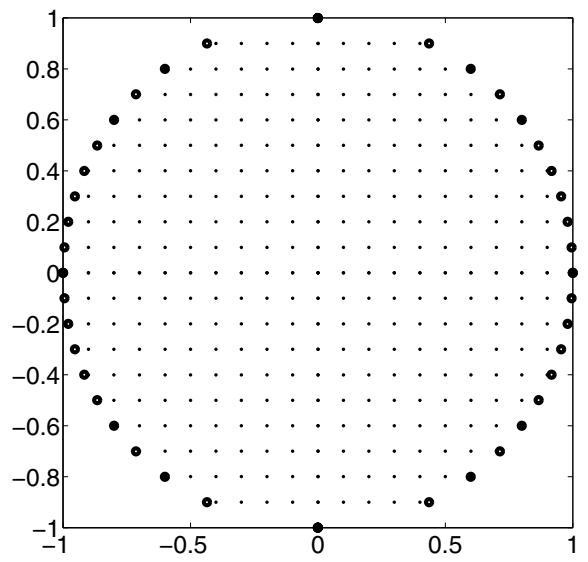

(b) $y$ sweep

Figure 5. Grids used for ADI splitting

Since the ADI method is used, the boundary conditions are enforced by constructing the integrals along each horizontal/vertical line on the mesh (small dots) in turn, with two additional points (large dots) which are on the circle. At these points, we enforce that $u$ vanish, by augmenting the boundary matrix $A_{h}^{d}$ (4.14) accordingly. As shown in Figure 4, no spurious effects at the boundary are observed, even though several points on the circle come arbitrarily close to the internal mesh.

\section{Conclusion}

Thus we have introduced a computational method for solving the wave equation with the method of lines transposed. Our approach differs from existing methods in that we solve the semi-discrete equation using a boundary integral solution. Since our method is implicit, there is no restriction on the time step, and so we have a competitive advantage over explicit time stepping algorithms.

We have considered a fully implicit scheme (i.e., in which the Helmholtz operator is treated implicitly), and showed that it is dissipative, as well as a time-averaged approach that leads to a non-dissipative scheme. We also analyzed the fully discretized schemes that result when the integral solution is discretized using the midpoint and trapezoidal rules. We showed convergence and stability for each of these schemes and validated our models with a numerical example.

\section{ACKNOWLEDGMENTS}

This work has been supported in part by AFOSR grants FA9550-11-1-0281, and FA9550-12-1-0455, and by NSF grant DMS-1115709.

\section{REFERENCES}

[1] Uri M. Ascher, Robert M. M. Mattheij, and Robert D. Russell, Numerical solution of boundary value problems for ordinary differential equations, Classics in Applied Mathematics, vol. 13, Society for Industrial and Applied Mathematics (SIAM), Philadelphia, PA, 1995. Corrected reprint of the 1988 original. MR1351005 (96f:65075) 
[2] H. Cheng, L. Greengard, and V. Rokhlin, A fast adaptive multipole algorithm in three dimensions, J. Comput. Phys. 155 (1999), no. 2, 468-498, DOI 10.1006/jcph.1999.6355. MR.1723309 (2000h:65178)

[3] R. Coifman, V. Rokhlin, and S. Wandzura, The fast multipole method for the wave equation: A pedestrian prescription, IEEE Trans. Antennas and Propagation 35 (1993), no. 3, 7-12.

[4] Zydrunas Gimbutas and Vladimir Rokhlin, A generalized fast multipole method for nonoscillatory kernels, SIAM J. Sci. Comput. 24 (2002), no. 3, 796-817 (electronic), DOI 10.1137/S1064827500381148. MR1950512(2004a:65176)

[5] L. Greengard and V. Rokhlin, A fast algorithm for particle simulations, J. Comput. Phys. 73 (1987), no. 2, 325-348, DOI 10.1016/0021-9991(87)90140-9. MR918448 (88k:82007)

[6] Ulrich Hornung, A parabolic elliptic variational inequality, Manuscripta Math. 39 (1982), no. 2-3, 155-172, DOI 10.1007/BF01165783. MR675536 (83m:49010)

[7] S.D. Jackson and P.H. Muir, Theory and numerical simulation of nth-order cascaded Raman fiber lasers, JOSA B 18 (2001), no. 9, 1297-1306.

[8] Jun Jia and Jingfang Huang, Krylov deferred correction accelerated method of lines transpose for parabolic problems, J. Comput. Phys. 227 (2008), no. 3, 1739-1753, DOI 10.1016/j.jcp.2007.09.018. MR2450970(2009k:65175)

[9] Leon Lapidus and George F. Pinder, Numerical solution of partial differential equations in science and engineering, A Wiley-Interscience Publication, John Wiley \& Sons Inc., New York, 1999. MR 1718868

[10] Peijun Li, Hans Johnston, and Robert Krasny, A Cartesian treecode for screened Coulomb interactions, J. Comput. Phys. 228 (2009), no. 10, 3858-3868, DOI 10.1016/j.jcp.2009.02.022. MR2511077(2010d:78012)

[11] Annamaria Mazzia and Francesca Mazzia, High-order transverse schemes for the numerical solution of PDEs, J. Comput. Appl. Math. 82 (1997), no. 1-2, 299-311, DOI 10.1016/S03770427(97)00090-3. 7th ICCAM 96 Congress (Leuven). MR1473548(98e:65072)

[12] N. Muthiyalu and S. Usha, Eigenvalues of centrosymmetric matrices (English, with German summary), Computing 48 (1992), no. 2, 213-218, DOI 10.1007/BF02310535. MR1169141 (93g:15008)

[13] Xiaodi Sun and Michael J. Ward, Dynamics and coarsening of interfaces for the viscous Cahn-Hilliard equation in one spatial dimension, Stud. Appl. Math. 105 (2000), no. 3, 203234, DOI 10.1111/1467-9590.00149. MR1783547(2001e:74069)

[14] Wen-Chyuan Yueh and Sui Sun Cheng, Explicit eigenvalues and inverses of tridiagonal Toeplitz matrices with four perturbed corners, ANZIAM J. 49 (2008), no. 3, 361-387, DOI 10.1017/S1446181108000102. MR2440234 (2009f:15023)

[15] A. Zafarullah, Application of the method of lines to parabolic partial differential equations with error estimates, J. Assoc. Comput. Mach. 17 (1970), 294-302. MR0280025 (43 \#5746)

Department of Mathematics, Michigan State University, East Lansing, Michigan 48824

E-mail address: causleym@math.msu.edu

Department of Mathematics, Michigan State University, East Lansing, Michigan 48824

E-mail address: christlieb@math.msu.edu

Institute for Cyber-Enabled Research, Michigan State University, East Lansing, MiCHIGAN 48824

E-mail address: ongbw@msu.edu

Department of Mathematics, Anderson University, Anderson, Indiana 46012

E-mail address: glvangroningen@anderson.edu 